\title{
Cirugía cardiaca pediátrica mínimamente invasiva: experiencia de un centro colombiano cardiovascular
}

\author{
Pediatric heart surgery minimally invasive: experience of a Colombian center
}

Keerby Hernández-Ruiz ${ }^{1 *}$, Diana Fajardo², Luis H. Díaz³, Miguel Ruz, Jorge Zapata ${ }^{5}$, Isabel Sánchez $z^{6}$ y Pablo Vásquez

${ }^{1}$ Servicio de Cardiología Pediátrica, Universidad Pontificia Bolivariana; ${ }^{2}$ Servicio de Cirugía Cardiaca, Clínica Cardio VID; ${ }^{3}$ Servicio de Cardiología Pediátrica Hemodinamista, Clínica Cardio VID; ${ }^{4}$ Servicio de Cardiología Pediátrica Intensivista, Clínica Cardio VID; ${ }^{5}$ Servicio de Cirugía Cardiovascular, Clínica Cardio VID; ${ }^{6}$ Servicio de Pediatría, Facultad de Medicina, Universidad Pontificia Bolivariana; ${ }^{7}$ Facultad de Pediatría, Universidad Nacional de Colombia. Medellín, Colombia

\section{Resumen}

Objetivo: Durante las últimas décadas, la cirugía cardiaca ha evolucionado, existe un interés creciente en minimizar la agresión corporal de procedimientos quirúrgicos, buscando una rápida recuperación, los abordajes mínimamente invasivos han sido tendencia en los últimos años, con seguridad y eficacia equivalente a las técnicas tradicionales de esternotomía. El objetivo de este estudio es comparar resultados clínicos entre pacientes pediátricos con cardiopatías congénitas sometidos a cirugía mínimamente invasiva frente a esternotomía estándar. Métodos: Estudio de casos y controles, anidado en una cohorte retrospectiva. Se incluyeron todos los pacientes pediátricos mayores de $10 \mathrm{~kg}$ de peso, sometidos entre 2014 a 2019 a corrección quirúrgica de cardiopatías congénitas simples, en un centro cardiovascular en Medellín. Se definió caso aquel que fuera sometido a abordaje mínimamente invasivo (MICS) y control los pacientes que se abordaron de manera convencional (CONV). Un total de 122 pacientes fueron admitidos, con una media de edad (MICS: 6 [4-12] vs. CONV: 5 [2-8 años]). No se encontraron diferencias en las características basales de ambos grupos. No se documentó diferencia estadísticamente significativa en los tiempos de perfusión (MICS: 67 min [50-90] vs. CONV 53 min: [42-90]; p=0,54), ni diferencias en las complicaciones (MICS: $7.4 \%$ vs. CONV: $8.8 \% ; p=0.77$ ). Los pacientes MICS tuvieron un lactato al ingreso y una estadía en la UCI menor que los controles. Conclusión: El abordaje MICS resulta ser una técnica novedosa, menos invasiva, segura y eficiente, frente al abordaje quirúrgico CONV, para la reparación de cardiopatías congénitas simples en paciente pediátricos.

Palabras clave: Cirugía torácica. Cardiopatías congénitas. Toracotomía. Esternotomía.

\begin{abstract}
Objective: During the last decades, cardiac surgery has revolutionized, there is a growing interest in minimizing the physical aggression of surgical procedures, seeking a quick recovery, minimally invasive approaches have been a trend in recent years, with safety and efficacy equivalent to traditional techniques sternotomy. The objective of this study is to compare clinical results between pediatric patients with congenital heart disease undergoing minimally invasive surgery versus standard sternotomy. Methods: Case-control study, nested in a retrospective cohort. All pediatric patients over $10 \mathrm{~kg}$ in weight were in-

\section{Correspondencia:}

*Keerby Hernández-Ruiz

E-mail: keerby.cardioped@gmail.com

Disponible en internet: 23-02-2021

Arch Cardiol Mex. 2022;92(1):19-25 www.archivoscardiologia.com

1405-9940 / @ 2021 Instituto Nacional de Cardiología Ignacio Chávez. Publicado por Permanyer. Este es un artículo open access bajo la licencia CC BY-NC-ND (http://creativecommons.org/licenses/by-nc-nd/4.0/).

Fecha de recepción: 11-08-2020

Fecha de aceptación: 19-01-2021 DOI: 10.24875/ACM.20000380
\end{abstract}


cluded, between 2014 and 2019 who underwent surgical correction of simple congenital heart disease, in a cardiovascular center in Medellin. A case was defined as one that underwent a minimally invasive approach (MICS) and control patients who were approached in a conventional manner (CONV). 122 patients were admitted, with a mean age (MICS: 6, 4-12 vs. CONV: 5, 2-8 years). No differences were found in the baseline characteristics of both groups. No statistically significant difference was documented in infusion times (MICS: 67 min [50-90] vs. CONV: 53 min [42-90]; $p=0.54$ ), nor differences in complications (MICS: 7.4 vs. CONV: 8.8\%; $p=0.77$ ). MICS patients had lactate on admission and a shorter ICU stay than controls. Conclusion: The MICS approach turns out to be a novel, less invasive, safe and efficient technique compared to the conventional surgical approach for the repair of simple congenital heart disease in pediatric patients.

Keywords: Thoracic surgery. Congenital heart disease. Thoracotomy sternotomy.

\section{Introducción}

Las primeras cirugías cardiacas mínimamente invasivas (MICS) se han usado en varios campos de la cirugía, sin embargo, en cirugía cardiaca su adopción tardó unos cuantos años más, debido a la preocupación con respecto a la canulación y el acceso limitado para cirugías grandes, con riesgo de embolia y dificultades para de airear las cavidades, por lo que se pensaba que sus resultados eran inferiores, sin embargo a partir de la década de 1990 han existido mejoras importantes en cuanto a la técnica, con desarrollo de circulación cardiopulmonar por medio de vasos periféricos, uso de sistemas de vacío, nuevos diseños de cánulas, mejores soluciones de cardioplejía y avances en la técnica de ecocardiografía transesofágica ${ }^{1}$.

Los abordajes incluidos en el concepto de mínima invasión son: minitoracotomía (anterior derecha, izquierda lateral, izquierda posterior), esternotomías parciales (incisión paraesternal, incisión transesternal, esternotomía superior, miniesternotomía en T, esternotomía en $T$ invertida y esternotomía en $L$ invertida). Los procedimientos quirúrgicos susceptibles a este abordaje incluyen: revascularización coronaria híbrida, implante de válvula aórtica transcatéter, cierre de defectos septales auriculares y ventriculares, y valvuloplastias, entre otros ${ }^{2-4}$.

El objetivo del presente trabajo fue comparar los resultados clínicos intrahospitalarios entre pacientes pediátricos admitidos a la unidad de cuidados intensivos en postoperatorio de corrección de cardiopatías congénitas abordables desde el lado derecho del corazón, que incluyen: defectos aislados septales, anomalías valvulares auriculoventriculares y conexiones venosas anómalas, que fueron sometidos a MICS frente a una corrección mediante abordaje convencional (CONV).

\section{Materiales y métodos}

Se trata de un estudio de casos y controles, anidado en una cohorte retrospectiva, en el cual se incluyeron todos los pacientes pediátricos mayores de $10 \mathrm{~kg}$ de peso, entre enero de 2014 y junio de 2019 que ingresaron a la unidad de cuidado intensivos en postoperatorio de corrección de defectos septales auriculares o ventriculares, anomalías valvulares auriculoventriculares aisladas o conexiones venosas anómalas parciales, con fisiología biventricular, en un centro cardiovascular en la ciudad de Medellín. Se excluyeron los pacientes con presencia de vena cava izquierda persistente, ductus arterioso o necesidad de intervención en la válvula pulmonar. Se definió caso aquel que fuera sometido a abordaje por MICS y control los pacientes que se abordaron de manera convencional por esternotomía mediana. La elección del tipo de abordaje se realizó en conjunto con los padres o cuidadores, después de evaluar las ventajas estéticas de la MICS.

Se realizó una revisión retrospectiva de los registros médicos con respecto a historia clínica y información perioperatoria, los datos fueron obtenidos de la base de datos de los servicios de cuidados intensivos pediátricos y de cirugía cardiovascular. Se analizaron parámetros demográficos como: edad, sexo, peso, parámetros del diagnóstico cardiológico y quirúrgicos como tiempos de pinzamiento aórtico, de bomba, vía de abordaje, canulación y tipo de procedimiento. Adicionalmente se recogieron variables hospitalarias como: estancia en unidad de cuidados intensivos (UCIP) y total hospitalaria, drenaje por sondas a tórax durante las primeras 24 horas, lactato al ingreso y a las 24 horas (y se calculó su aclaramiento). Se midió la necesidad de transfusión de hemoderivados, complicaciones y muerte.

El estudio fue aprobado por el Comité de Ética de Investigaciones en Salud de la Universidad Pontificia Bolivariana y de la Clínica Cardio VID (Medellín, CoIombia), y fue diseñado y llevado a cabo teniendo en 
cuenta salvaguardar los principios éticos de beneficencia, no maleficencia, respeto y justicia.

\section{Protocolo quirúrgico}

Todos los pacientes sometidos a corrección de cardiopatías congénitas son evaluados previamente por un equipo multidisciplinario del staff médico quirúrgico en nuestra institución. Los pacientes considerados aptos para manejo quirúrgico y que requieren circulación extracorpórea son admitidos con estudios complementarios que incluyen analítica sanguínea, radiografía de tórax y electrocardiograma. Se requiere el consentimiento informado por parte de sus padres o cuidadores. En salas de cirugía, previa asepsia y antisepsia, bajo anestesia general y cuidado de anestesiología cardiovascular, se realiza una canulación periférica a través de los vasos femorales para establecer el bypass cardiopulmonar total; bajo guía ecográfica se realiza canulación directa de la cava superior, se realiza un abordaje, ya sea por minitoracotomía axilar, minitoracotomía lateral o periareolar, y posteriormente disección por planos hasta cavidad pleural; se realiza pericardiotomía y puntos de reparo en el pericardio. Se inicia circulación extracorpórea, se instauran torniquetes en ambas cavas. Se realiza pinzamiento aórtico, cardioplejía sanguínea anterógrada fría tipo del Nido por raíz aórtica. Atriotomía derecha, visualización del defecto y reparo de este. Cierre de aurícula derecha, previas maniobras de desaireación, se retira pinza aórtica. Destete lento de circulación extracorpórea. Al final del procedimiento se realiza una evaluación ecocardiográfica transesofágica con el fin de evaluar el éxito del procedimiento y la necesidad de reintervención. Se realiza ultrafiltración modificada, revisión y control de hemostasia, descanulación, reversión de la anticoagulación con protamina y se dejan sondas a pleura y en mediastino, uno o dos electrodos en epicardios auricular y ventricular, según cada caso particular, para la monitorización de alteraciones del ritmo cardiaco. Se realiza cierre de pericardio parcial, infiltración intercostal con bupivacaína, cierre de cavidad pleural, cierre por planos de piel y de herida inguinal.

\section{Análisis estadísticos}

La información fue tabulada en Excel y analizada en Stata 13, luego de verificar datos incompletos y extremos. Para la estadística descriptiva para las variables categóricas se informaron frecuencia y porcentaje, y las variables continuas que no tenían una distribución normal fueron presentadas con mediana (p50) y percentiles intercuartiles (p25, p75). Los casos se compararon a los controles por una regresión logística simple y se presentan odds ratios (OR), intervalos de confianza del 95\% (IC 95\%) y estadístico de prueba (p). Este protocolo fue presentado al comité de ética de la institución, que autorizó su realización con excepción de consentimiento informado por su naturaleza.

\section{Resultados}

Un total de 122 pacientes fueron admitidos en la UCIP en postoperatorio de corrección de patologías abordables a través de la aurícula derecha como: comunicación interauricular, interventricular, estenosis o insuficiencia mitral o tricuspídea aisladas y anomalías en las conexiones venosas. Entre los pacientes, 54 fueron sometidos a MICS y 68 a esternotomía convencional. La tabla 1 muestra las características demográficas de la población. El defecto cardiaco predominante fue la comunicación interauricular (CIA). Las cardiopatías congénitas sometidas a MICS se encuentran detalladas en la figura 1. Se encontró un caso de síndrome de cimitarra, en el cual se realizó corrección de comunicación anómala venosa pulmonar y creación de túnel intracardiaco. Los tiempos de perfusión y de pinzamiento aórtico, aunque fueron menores en el grupo control, no mostraron significancia estadística entre los grupos.

Los procedimientos realizados por MICS y su frecuencia se encuentran detallados en la tabla 2. El abordaje preferido en el grupo de MICS fue toracotomía axilar, seguida de toracotomía lateral derecha y periareolar, la canulación se realizó preferiblemente en el grupo MICS vía periférica a través de los vasos arterial y venoso femoral, en el grupo CONV se realizó un abordaje mediante toracotomía mediana convencional. Al ingreso a UCIP se encontró un lactato inicial significativamente menor en los pacientes MICS, sin embargo, la diferencia y la depuración en las primeras 24 horas fue similar. Los drenajes a través de las sondas a tórax las primeras 24 horas fueron menores en los pacientes de MICS. No hubo diferencias en el requerimiento de transfusión de hemoderivados ni en la tasa de complicaciones. Las complicaciones postoperatorias se presentaron en un $8.8 \%$. Sucedieron en cuatro pacientes sometidos a MICS y fueron: un paciente con bajo gasto postoperatorio, uno con derrame pleural con resolución a los tres días, disfunción del nodo sinusal en un paciente asintomático y en uno se documentó parálisis hemidiafragmática sin afectar su 
Tabla 1. Análisis comparativo de cirugía mínimamente invasiva frente a cirugía convencional

\begin{tabular}{|c|c|c|c|c|c|c|c|c|}
\hline & MICS & $\mathrm{n}=54$ & CONV & $n=68$ & $\mathrm{OR}^{*}$ & $\mathbf{p}$ & \multicolumn{2}{|c|}{ IC 95\% } \\
\hline $\begin{array}{l}\text { Demográficas } \\
\text { Edad (años), p50 (p25-p75) } \\
\text { Peso (kg), p50 (p25-p75) } \\
\text { Sexo femenino, n \% }\end{array}$ & $\begin{array}{c}6 \\
21.0 \\
37\end{array}$ & $\begin{array}{c}(4-12) \\
(15.6-35) \\
68.5 \%\end{array}$ & $\begin{array}{c}5 \\
16.8 \\
43\end{array}$ & $\begin{array}{c}(3-8) \\
(13.3-21.1) \\
63.2 \%\end{array}$ & $\begin{array}{l}1.10 \\
1.03 \\
1.27\end{array}$ & $\begin{array}{l}0.038 \\
0.042 \\
0.542\end{array}$ & $\begin{array}{l}1.01 \\
1.00 \\
0.59\end{array}$ & $\begin{array}{l}1.19 \\
1.06 \\
2.70\end{array}$ \\
\hline $\begin{array}{l}\text { Diagnóstico cardiaco, n \% } \\
\text { CIA } \\
\text { Anomalías valvulares } \\
\text { Otros }\end{array}$ & $\begin{array}{c}47 \\
5 \\
2\end{array}$ & $\begin{array}{l}87.0 \% \\
9.3 \% \\
3.7 \%\end{array}$ & $\begin{array}{c}57 \\
8 \\
3\end{array}$ & $\begin{array}{l}83.8 \% \\
11.8 \% \\
4.4 \%\end{array}$ & $\begin{array}{l}\text { (Ref.) } \\
0.76 \\
0.81\end{array}$ & $\begin{array}{l}0.646 \\
0.820\end{array}$ & $\begin{array}{l}0.23 \\
0.13\end{array}$ & $\begin{array}{l}2.47 \\
5.04\end{array}$ \\
\hline $\begin{array}{l}\text { Quirúrgicas } \\
\text { Minutos, p50 (p25-p75) }\end{array}$ & & & & & & & & \\
\hline $\begin{array}{l}\text { Perfusión } \\
\text { Pinza } \\
\text { Lactato mmol/l, p50 (p25-p75) }\end{array}$ & $\begin{array}{l}67 \\
32\end{array}$ & $\begin{array}{l}(50-90) \\
(24-50)\end{array}$ & $\begin{array}{c}53 \\
26.5\end{array}$ & $\begin{array}{c}(42-90) \\
(21.0-46.5)\end{array}$ & $\begin{array}{l}1.00 \\
1.00\end{array}$ & $\begin{array}{l}0.545 \\
0.700\end{array}$ & $\begin{array}{l}0.99 \\
0.99\end{array}$ & $\begin{array}{l}1.01 \\
1.02\end{array}$ \\
\hline $\begin{array}{l}\text { Ingreso } \\
24 \text { horas }\end{array}$ & $\begin{array}{l}2.1 \\
1.2\end{array}$ & $\begin{array}{l}(2.0-3.0) \\
(0.9-1.4)\end{array}$ & $\begin{array}{l}3.0 \\
1.2\end{array}$ & $\begin{array}{c}(2.3-4.2) \\
(1-1.7)\end{array}$ & $\begin{array}{l}0.53 \\
0.58\end{array}$ & $\begin{array}{l}0.001 \\
0.084\end{array}$ & $\begin{array}{l}0.36 \\
0.32\end{array}$ & $\begin{array}{l}0.77 \\
1.08\end{array}$ \\
\hline Delta \% & $52.2 \%$ & $(40.0-56.5 \%)$ & $59.2 \%$ & $(30.1-69.0 \%)$ & 1.76 & 0.375 & 0.50 & 6.15 \\
\hline Trasfusiones, n \% & 4 & $7.4 \%$ & 6 & $8.8 \%$ & 0.83 & 0.777 & 0.22 & 3.09 \\
\hline Complicaciones, n \% & 4 & $7.4 \%$ & 6 & $8.8 \%$ & 0.83 & 0.777 & 0.22 & 3.09 \\
\hline $\begin{array}{l}\text { Drenaje sondas ml/24 h, p50 (p25-p75) } \\
\text { Desenlaces } \\
\text { Estadía (días), p50 (p25-p75) }\end{array}$ & 118 & (75-170) & 133 & (90-185) & 1.00 & 0.074 & 0.99 & 1.00 \\
\hline $\begin{array}{l}\text { UCIP } \\
\text { Hospital }\end{array}$ & $\begin{array}{l}1 \\
4\end{array}$ & $\begin{array}{l}(1-1) \\
(4-5)\end{array}$ & $\begin{array}{l}2 \\
4\end{array}$ & $\begin{array}{l}(2-3) \\
(4-5)\end{array}$ & $\begin{array}{l}0.11 \\
0.99\end{array}$ & $\begin{array}{l}0.000 \\
0.827\end{array}$ & $\begin{array}{l}0.05 \\
0.88\end{array}$ & $\begin{array}{l}0.24 \\
1.11\end{array}$ \\
\hline
\end{tabular}

*OR crudo por regresión logística simple.

MICS: cirugía mínimamente invasiva; CONV: estereotomía media convencional; CIA: comunicación interauricular; UCIP: unidad de cuidados intensivos pediátricos; OR: odds ratio.

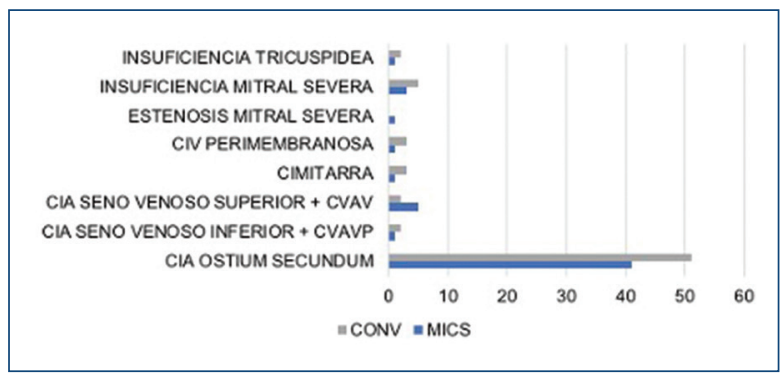

Figura 1. Cardiopatías congénitas abordadas. CIA: comunicación interauricular; CVAP: conexión venosa pulmonar anómala parcial; CIV: comunicación interventricular; CONV: estereotomía convencional; TOR: esternotomía media convencional.

dinámica ventilatoria; en el grupo CONV se presentaron complicaciones en seis pacientes, tres de ellos con bajo gasto postoperatorio, dos presentaron derrame pleural y en uno de ellos se documentó bloqueo auriculoventricular transitorio. Ningún caso requirió conversión a esternotomía, no se presentaron fallecimientos durante este periodo de tiempo. Durante el seguimiento ambulatorio los resultados estéticos fueron mejores en los pacientes con abordaje MICS (Fig. 2) y no se documentó infección en el sitio operatorio en nuestra cohorte.

\section{Discusión}

Las cardiopatías congénitas no complicadas, como defectos de los tabiques auricular y ventricular o lesiones valvulares auriculoventriculares aisladas tienen tasas muy altas de éxito tras el reparo quirúrgico convencional mediante estereotomía mediana, sin embargo, los resultados estéticos pueden ser desfavorables, alterando la imagen corporal, pudiendo llegar a generar dificultades psicológicas y sociales secundario a los problemas de aceptación de los cambios corporales $^{5}$. En este sentido, las cicatrices son procesos que implican cambios sustanciales a nivel estético especialmente en los niños y adolescentes que se enfrentan a un periodo de transición psicosomática. Los procesos que requieren una intervención quirúrgica a menudo son vistas como una alteración corporal, la cual requiere un proceso de afrontamiento por parte del paciente y su familia ${ }^{6}$. Es por esto por lo cual cada vez más crece el interés de ofrecer a nuestros pacientes 
Tabla 2. Procedimientos realizados a través de MICS

\begin{tabular}{|l|c|c|}
\hline Procedimiento & MICS & $\mathbf{n}=\mathbf{5 4}$ \\
\hline Cierre de CIA por parche & 41 & $76 \%$ \\
\hline Cierre de CIA seno venoso superior y CVAP & 3 & $5.6 \%$ \\
\hline Cierre de CIV por parche & 1 & $1.9 \%$ \\
\hline Cirugía de Warden & 1 & $1.9 \%$ \\
\hline Corrección de CIA seno venoso inferior & 1 & $1.9 \%$ \\
\hline Plastia mitral & 4 & $7.4 \%$ \\
\hline Plastia tricúspidea & 1 & $1.9 \%$ \\
\hline $\begin{array}{l}\text { Corrección de drenaje venoso + creación de } \\
\text { túnel intraventricular }\end{array}$ & 1 & $1.9 \%$ \\
\hline $\begin{array}{l}\text { Reemplazo de válvula mitral por prótesis } \\
\text { mecánica }\end{array}$ & 1 & $1.9 \%$ \\
\hline
\end{tabular}

MICS: mínimamente invasivo; CIA: comunicación interauricular; CVAP: conexión venosa pulmonar anómala parcial; CIV: comunicación interventricular.

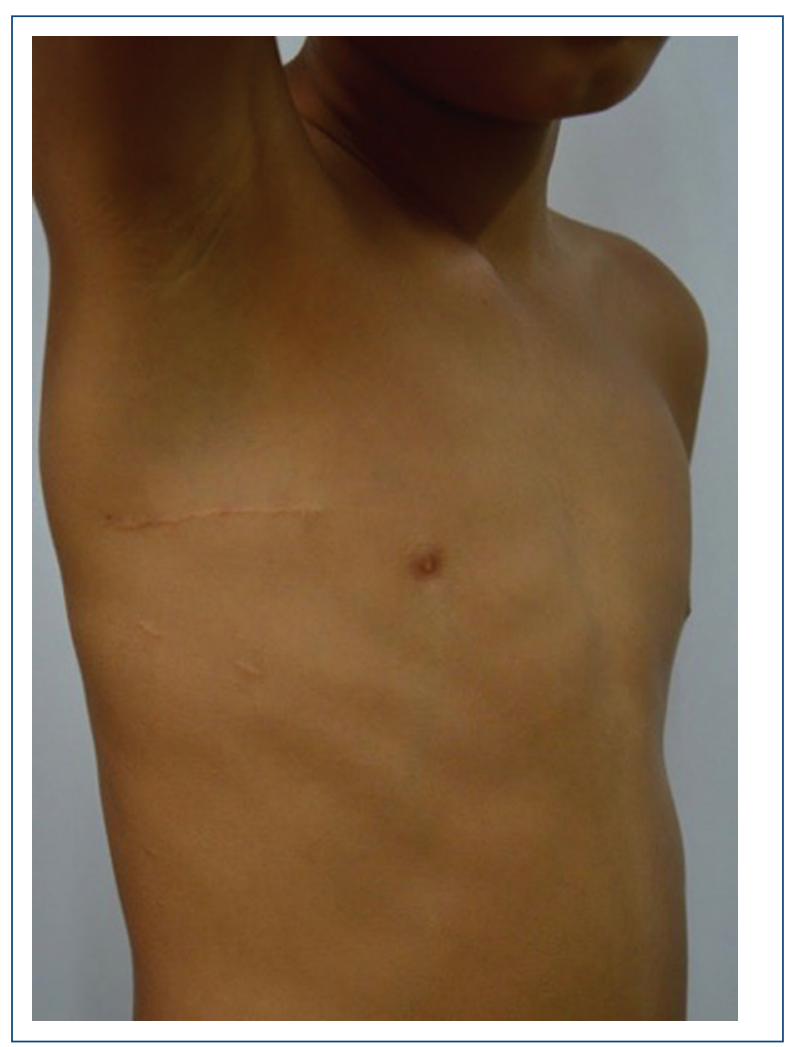

Figura 2. Resultado estético posterior a una cirugía cardiaca mediante un abordaje mínimamente invasivo.

los mejores resultados evaluados bajo una óptica de integralidad.

Mantener los altos estándares de calidad independiente del tipo de abordaje permite lograr buenos resultados en el procedimiento sin incrementar los riesgos. Los beneficios descritos de MICS en centros experimentados han sido múltiples, al compararlos con la esternotomía clásica, se encuentran ventajas en cuanto a una recuperación pulmonar más rápida, menor tiempo de ventilación mecánica, disminución de la estancia en UCIP, menor requerimiento de hemoderivados y mejor resultado estético ${ }^{7,8}$. En nuestra experiencia el abordaje por minitoracotomía fue suficiente para una adecuada exposición quirúrgica, permitiendo la corrección de los defectos y un desenlace exitoso en la totalidad de los casos, sin necesidad de reintervención o evidencia de lesiones residuales significativas en la evaluación ecocardiográfica postoperatoria.

El objetivo es realizar la misma técnica de reparación de la cardiopatía por medio de un abordaje alternativo, menos invasivo. El abordaje preferido en nuestra cohorte fue la toracotomía axilar derecha, seguida de toracotomía lateral derecha y periareolar; la decisión de la variante se realizó con base en el defecto anatómico a reparar. En el estudio de 15 años de experiencia acumulada de abordaje MICS destacó que la introducción de diferentes accesos alternativos permite establecer pautas de aprendizaje que posteriormente facilitan la selección del abordaje en función de la cardiopatía y la relación pondoestatural del niño9.

En nuestro estudio, similar a lo que se encuentra descrito en la literatura actual, encontramos menor tiempo de estancia en UCIP y de drenaje a través de las sondas a tórax las primeras 24 horas, así mismo el lactato a su ingreso fue menor, el cual podría soportar un menor estrés hemodinámico durante el abordaje mínimamente invasivo, con menor lesión tisular muscular y de tejidos blandos, todo esto favoreciendo una recuperación más temprana. No encontramos diferencias con significancia estadística relacionadas con los tiempos quirúrgicos (tiempo de perfusión y tiempo de bomba), lo cual sugiere que en centros experimentados esta variable no se presentaría como una objeción para realizar un abordaje mínimamente invasivo.

La MICS no se asoció con un incremento en la morbilidad operatoria o postoperatoria, no se documentó ninguna complicación relacionada con la herida quirúrgica, las complicaciones se presentaron en similar porcentaje tanto en los casos como en los controles y estuvieron relacionadas con trastornos en el ritmo como disfunción sinusal y un caso de bloqueo auriculoventricular transitorio en un paciente con abordaje convencional que pudiera estar condicionada por el defecto cardiaco anatómico y sus relaciones con el tejido de conducción. Se documentó derrame pleural 
derecho y un caso de parálisis hemidiafragmática, en los que se realizó seguimiento clínico por su nulo impacto en la dinámica ventilatoria de los pacientes; en total cuatro pacientes presentaron bajo gasto postoperatorio, tres de ellos en el grupo CONV, sin embargo, las múltiples variables que implica el desarrollo de esta complicación no permiten realizar una relación solamente con la variante de abordaje quirúrgico.

No se presentó ningún caso de mortalidad. Los estudios publicados de mortalidad en ambas técnicas son similares hasta la fecha, excepto en los pacientes de alto riesgo, en quienes se ha demostrado una reducción en la mortalidad con MICS ${ }^{10-13}$; igualmente se ha documentado en la literatura menor mortalidad precoz y tardía, principalmente en las cirugías de recambio valvular aórtico, así como un menor sangrado postoperatorio y menor requerimiento de hemoderivados ${ }^{14-19}$. En un estudio de cohortes ajustadas por nivelación del riesgo de propensión, demostró que las técnicas de reparo y sustitución de la válvula mitral a través de toracotomía derecha pueden considerarse un procedimiento seguro y eficaz corto y largo plazo ${ }^{20}$.

Específicamente en la población pediátrica se han realizado estudios en cardiopatías congénitas estructurales por medio de miniesternotomías y minitoracotomías derechas con excelentes resultados quirúrgicos y cosméticos, principalmente en cardiopatías congénitas de bajo riesgo como en defectos septales; pero también se han realizado en casos más complejos, como CIA tipo seno venoso, CIA ostium primum, defecto septal atrioventricular parcial, prótesis mitral e incluso tracto de salida izquierdo (membrana subaórtica) y tetralogía de Fallot ${ }^{21-25}$. Existen estudios que documentan sus beneficios en cuanto a cierre de fístula de arteria coronaria, evidenciando una excelente exposición quirúrgica en pacientes seleccionados ${ }^{26}$. De cualquier manera, a pesar de que los resultados parecen ser favorables, debe iniciarse con cirugías de baja complejidad, abordables a través de la aurícula derecha y posteriormente ir avanzando en patologías más complejas, una vez se sobrepase la curva de aprendizaje.

Al valorar los predictores de mortalidad evidenciados en publicaciones recientes, se extrapolan datos de estudios realizados en población adulta, en quienes se consideran factores predictores el EuroSCORE, el péptido natriurético tipo $\mathrm{B}$ y la escala funcional de la New York Heart Association, además también se menciona el tiempo de circulación extracorpórea y el tiempo de pinzamiento aórtico ${ }^{27}$. Cabe aclarar que los resultados son mejores en pacientes seleccionados y tratados en centros hospitalarios con un alto volumen de pacientes sometidos a cirugía cardiaca y en manos de cirujanos expertos en la técnica quirúrgica.

En la actualidad el abordaje MICS para cardiopatías congénitas no complejas se realiza obteniendo resultados excelentes, cada vez más, se consideran los efectos estéticos y psicológicos de la cicatrización en el grupo de población pediátrica, cada variante puede presentar ventajas y desventajas dependiendo del tipo de cardiopatía que tratar y la anatomía del paciente; se han descrito: mal desarrollo de la columna por afectarse la musculatura espinal en los casos de toracotomía posterior o alteraciones en la glándula mamaria en el caso de abordaje periareolar, actualmente se está trabajando en la mejora de las técnicas con el fin de obviar dichas deformidades futuras ${ }^{28}$.

La realización de toracotomía se ha considerado más dolorosa que la propia esternotomía por lo que el uso de analgésicos puede ser un factor diferenciador, a pesar de que es difícil encontrar diferencias objetivas en este tipo de comparaciones, en la actualidad en nuestra institución se está implementando una herramienta que permita objetivar las diferencias en este campo entre las dos técnicas.

Un aspecto fundamental que tener en cuenta en el momento de realizar una técnica mínimamente invasiva es que requiere un diagnóstico preciso, ya que este acceso no permite la exploración completa de las estructuras cardiacas, sin embargo, en la actualidad se cuenta con técnicas de imagen cardiovascular no invasiva que permiten un adecuado diagnóstico y planeación previo al acto quirúrgico.

\section{Conclusión}

La MICS resulta ser una técnica novedosa, que se presenta como una alternativa menos invasiva, con un perfil de seguridad similar y correcciones apropiadas, frente al abordaje quirúrgico convencional, para la reparación de defectos cardiacos congénitos simples, abordables por el corazón derecho en paciente pediátricos, intervenidos en centros con experiencia en el tratamiento de cardiopatías congénitas.

\section{Limitaciones del estudio}

Las limitaciones de nuestro estudio incluyen el número reducido de pacientes pediátricos incluidos, ya que obedece a una técnica relativamente nueva en nuestro medio, y aquellas inherentes a un estudio retrospectivo, porque existen algunas preguntas clínicas 
relacionadas con las técnicas mínimamente invasivas, como la evaluación objetiva del dolor, tiempos de extubación y evaluación de escalas de percepción corporal que no fueron explorados en este estudio.

\section{Financiamiento}

Ninguno.

\section{Conflicto de intereses}

Los autores declaran que no tienen ningún conflicto de intereses.

\section{Responsabilidades éticas}

Protección de personas y animales. Los autores declaran que para esta investigación no se han realizado experimentos en seres humanos ni en animales.

Confidencialidad de los datos. Los autores declaran que han seguido los protocolos de su centro de trabajo sobre la publicación de datos de pacientes.

Derecho a la privacidad y consentimiento informado. Los autores declaran que en este artículo no aparecen datos de pacientes.

\section{Bibliografía}

1. Bautista V. Cirugía de mínima invasión. Cir Cardiov. 2014;21(2):107-10.

2. Jaramillo JS, Matar OA. Presente y futuro en la cirugía cardiaca mínimamente invasiva. Rev Colomb Cardiol. 2013;20(4):259-61.

3. Santana O, Larrauri MC, Escolar E, Brenes JC, Lamelas J. La cirugía valvular mínimamente invasiva. Rev Colomb Cardiol. 2014;21(3):188-94

4. Gil-Jaurena JM, González-López MT, Pérez-Caballero R, Pita A, Castillo R, Miró L. Años de cirugía cardiaca infantil miniinvasiva; evolución y tendencias. An Pedatr (Barc). 2016;84(6):304-10.

5. Bolton MA, Lobben I, Stern TA. The impact of body image on patient care. Prim Care Companion J Clin Psychiatry. 2010;12(2):PCC.10r00947.

6. Ingoz F, Tavlasoglu M, Sahin MA, Kurkluoglu M, Guler A, Günay C, et al. Minimally invasive pediatric surgery in uncomplicated congenital hear disease. Asian Cardiovasc Thorac Ann. 2013;21(4):414-7.

7. Santana O, Reyna J, Grana R, Buendia M, Lamas GA, Lamelas J. Outcomes of minimally invasive valve surgery versus standard sternotomy in obese patients undergoing isolated valve surgery. Ann Thorac Surg. 2011;91:406-10.

8. González López MT, Gil Jaurena JM, Pita Fernández AM, Pérez Caballero-Martínez R, Rodríguez Ogando A, Álvarez Martín T, et al. 7002-9- Cirugía cardiaca pediátrica mínimamente invasiva: ¿cuándo, cómo y por qué? Experiencia inicial con 100 casos. Rev Esp Cardiol. 2015;68(Supl 1):43.
9. Gil-Jaurena JM, González-López MT, Pérez-Caballero R, Pita A, Castillo R, Miró L. 15 years of minimally invasive paediatric cardiac surgery; development and trends. An Pediatr (Barc). 2016;84(6):304-10.

10. Scarci $M$, Young $C$, Fallou $\mathrm{H}$. Is ministernotomy superior to conventional approach for aortic valve replacement? Interact Cardiovasc Thorac Surg. 2009;9:314-7.

11. Rodríguez EA, Otero J, Mataró MJ, Sánchez Espín G, Porras C Guzón A, et al. Cirugía valvular aórtica mínimamente invasiva. Cir Cardiov. 2016;23(6):306-11.

12. Chen DCH, Martin J, Lal A, Diegeler A, Folliguet TA, Nifong W, et al. Minimally invasive versus conventional open mitral valve surgery. A meta-analysis and systematic review. Innovations. 2011;6:84-103.

13. Gil-Jaurena JM, Zabala JI, Conejo L, Cuenca V, Picazo B, Jiménez C, et al. Cirugía mínimamente invasiva en niños. Corrección de la comunicación interauricular por vía axilar y submamaria. Rev Esp Cardiol. 2011;64(3):208-12.

14. Schmitto JD, Mokashi SA, Cohn LH. Minimally invasive valve surgery. J Am Coll Cardiol. 2010;56:455-62.

15. Paredes F, García-Fuster R, Sánchez MH, Villegas C, Hornero F, Gil Ó, et al. Abordaje mínimamente invasivo para el recambio valvular aórtico: ¿está asociado a menor transfusión de hemoderivados? Cir Cardiov. 2015;22(5):230-5.

16. Corona Perezgrovas MA, Sagahón Martínez JA, Hernández Mejía BI, Martínez Hernández HJ, Herrera Alarcón V. Abordaje mínimamente invasivo versus estereotomía total en la sustitución valvular aórtica: estudio comparativo de la evolución posoperatoria temprana. Cir Cardiov. 2015;22(5):224-9.

17. García Vieites M, Fernández Arias L, Portela F, García Barreiro J, Cuenca JJ, Bautista-Hernández V. Resultados de la implementación de un programa de cirugía mínimamente invasiva para cardiopatías congénitas estructurales. Cirugía Cardiov. 2012;19(2):194.

18. Chang C, Raza S, Altarabsheh SE, Delozier S, Sharma UM, Zia A, et al. Minimally Invasive aproaches to surgical aortic valve replacement: $A$ meta-analysis. Ann Thorac Surg. 2018;106:1881-9.

19. Rodríguez-Caulo EA, Otero Forero JJ, Mataró MJ, Sánchez Espín G, Porras C, Guzón A, et al. Melero Mejora de la morbilidad postoperatoria en recambio valvular aórtico aislado con miniesternotomía: estudio pareado por puntuación de propensión. Cir Cardiov. 2016;23(5):229-33.

20. Carmona García P, Errando Oyonarte C, Vázquez A, Cánovas S, Zarragoicoechea I. La cirugía mínimamente invasiva de la válvula mitral a través de toracotomía derecha es un procedimiento seguro y eficaz corto y largo plazo. Estudio de cohortes ajustadas por nivelación del riesgo de propensión. Rev Colomb Cardiol. 2016;23(6):535-44.

21. Borger MA, Moustafine V, Conradi L, Knosalla C, Richter M, Merk DR, et al. A randomized multicenter trial of minimally invasive rapid deployment versus conventional full sternotomy aortic valve replacement. Ann Thorac Surg. 2015;99:17-25.

22. Schumer EM, Slaughter MS. Cirugía mínimamente invasiva para la implantación de dispositivos de asistencia ventricular: ¿Es siempre recomendable? Rev Esp Cardiol. 2018;71(1):2-3.

23. García-Vieites M, Bautista-Hernández V, Cardenas I, Barreiro J, Loyola H, García I, et al. Miniesternotomía inferior. Revisión de los resultados quirúrgicos y cosméticos en nuestros 100 primeros casos. Cir Cardiov. 2015;22(3):129-34.

24. Gil JM, Zabala Jl. Cirugía cardiaca mínimamente invasiva en el niño. Cardiocore. 2013;48(1):17-20.

25. Del Nido PJ. Cirugía mínimamente invasiva para la comunicación interauricular en niños. Rev Esp Cardiol. 2011;64(3):177-8.

26. Song L, Dong Y, Wang B, Li J, Qi X, Gao X, et al. The evolution of the minimally invasive approach and conventional median sternotomy for coronary artery fistula correction. Heart Lung Circ. 2019;28:1277-82.

27. Lotz-Esquivel S, Matarrita-Quesada B, Monge-Bonilla C, Kuhn-Delgadillo K. Cirugía cardiaca en Costa Rica: Caracterización de los pacientes en el Hospital San Juan de Dios del 2010 al 2015. Cir Cardiov. 2019;26(2):71-80.

28. Giamberti A, Mazzera E, Di Chiara E, Ferretti, Pasquini L, Di Donato RM. Right submammary minithoractomy for repair of congenital heart defects. Eur J Cardiothorac Surg. 2000;18:678-82. 